\title{
Cascading of Air Quality Detector and Digital Data Transmission with Zero Error in Minimum Duration
}

\author{
Anitha $\mathrm{R}^{\mathrm{a}, 1}$, Veerasundaram $\mathrm{M}^{\mathrm{a}}$, Rajarajan $\mathrm{S}^{\text {a }}$, Jaya Prakash E ${ }^{\mathrm{a}}$, NandhaKishore ${ }^{\mathrm{a}}$, \\ Ajay Krishnan S R ${ }^{\text {a }}$ \\ a Department of EEE, Sri Sai Ram Institute of Technology, Chennai, TN, India
}

\begin{abstract}
The paper proposes a system to monitor the environment air quality using an Arduino microcontroller, to enhance the quality IOT Technology is used. The utilization of IoT technology improves the method of monitoring various factors of the environment issues. The MQ- 02 gas sensor is used to detect the various gases which are harmful to humans. A Wi-Fi module connects the whole system to the internet and an LCD is employed for the visual Output. This Automated Air management system may be a breakthrough to contribute an answer to the most important threat. The air quality detector overcomes the problems of the highly polluted areas which may be a major issue. This technique has features for the user to monitor the quantity of pollution on their smart devices using the appliance.
\end{abstract}

Keywords. IOT, Automatic Air management system, Electronic Image.

\section{Introduction}

Manual Scavenging is the most nauseating thing to try to do, for others, it's the sole thanks to making a living. Many manual scavengers die, asphyxiated by poisonous gases. A sewage system is a collection of underground pipes connected from every residential and commercial building to the treatment plant. from there the water is let into water bodies after treatment of sewage water. Due to improper care of more than thousand workers who works in manhole dies every year due to harmful gases like carbon monoxide, methane, hydrogen sulphide etc, [1] present inside the manhole. These gases need to keep up the track so that huge rise within the normal of effluents level should be known and corrective measures are often taken. On the contrary, the prevailing systems available aren't much portable and aren't affordable. Also, implementation is hard. The designed rover detects the presence of harmful gas in the monitoring system. The device consists of a processing section which takes input, processes and gives output [2]. This technique requires a base station with almost all the sensors.

In this project, an embedded system is meant with an Arduino Microcontroller and various gas sensors for the aim of monitoring, detecting and altering that helps in eliminating the lives of endangered humans [3]. The system is economical to implement and well-defined. within the existing system, a variety of jobs related to gas detection and ensuring security systems. It's been implemented among and a few were

${ }^{1}$ Anitha R, Department of EEE, Sri Sai Ram Institute of Technology, TN, India, Email: anitha.eee@sairamit.edu.in. 
demonstrated in practice to detect the harmful gas but both approaches were effective gas sensing units have been implemented which is capable of detecting the harmful and toxic gases individually and display in the LCD. Harmful gases like carbon monoxide, Methane, Hydrogen Sulphide these gases are very toxic to the human, Heart Beat sensor this may be fixed on the worker's hand watch and the message is going to be sent to outside workers and Municipal Officers with the assistance of IoT [4].

\section{Embedded System and IoT}

\subsection{Embedded System}

An embedded system is designed to perform one or limited set of functions using a combination of software and hardware. It adds intelligence to operation. The characteristics of an embedded system are i)Should have small set of functions ii)Low power consumption since it uses battery iii)Limited memory \&amp; Limited peripherals iv)Application predefined v)Not accessible directly vi)Highly reliable \&amp; need to operate with time constraints The simplest model consists of a processor, sensors, actuators and memory [5].

This solves any real time problems. Sensor senses the physical change and send it to the processor which is connected with the memory and actuators. The process of translating the code that is written by humans to the code understandable by machine is called the build process [6]. A hardware designer must take into account following features 1. Operation at higher clock speed 2. High computing performance [Pipelining, Prefetch cache and RISC Architecture 3. Fast context switching in Multitasking system. 4. Power efficient embedded system 5. Burst mode access 6 . Automatic operation for hardware solution to shared data problem [7-10].

\subsection{IoT}

IOT(Internet Of Things) is a highly distributed network of smart devices embedded with electronic sensors and software each capable of dynamically generating, analyzing and communicating intelligence that can be used to increase experimental efficiency and power and make life more easier and comfortable is shown in Figure 1 [11].

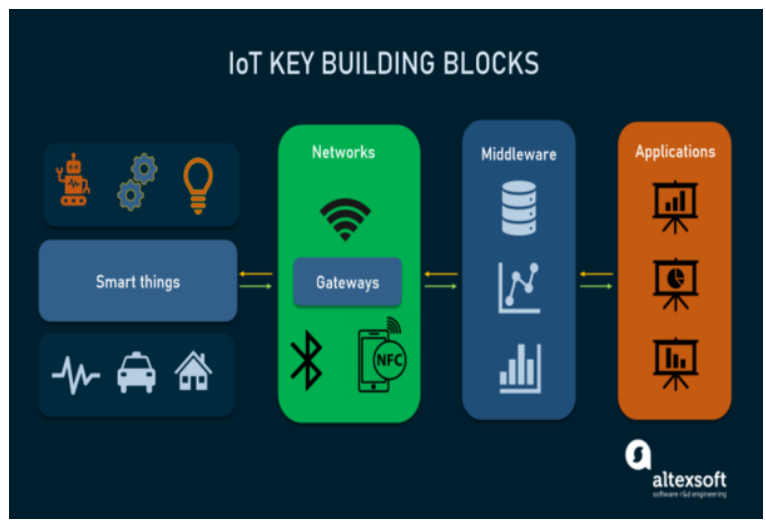

Figure 1. Structure of IOT 
IOT devices can collect various types of data through the onboard sensors or attached sensors such as Temperature, Humidity and Gas sensor.

\section{Existing System}

In existing systems which were mostly used in industrial areas to detect the leakage of harmful gas around them and alert the user, which is large, firm and complex in circuit design and cannot be used in homes, manhole, tunnels to analyse the atmosphere of that working areas [12].

\section{Flow Diagram}

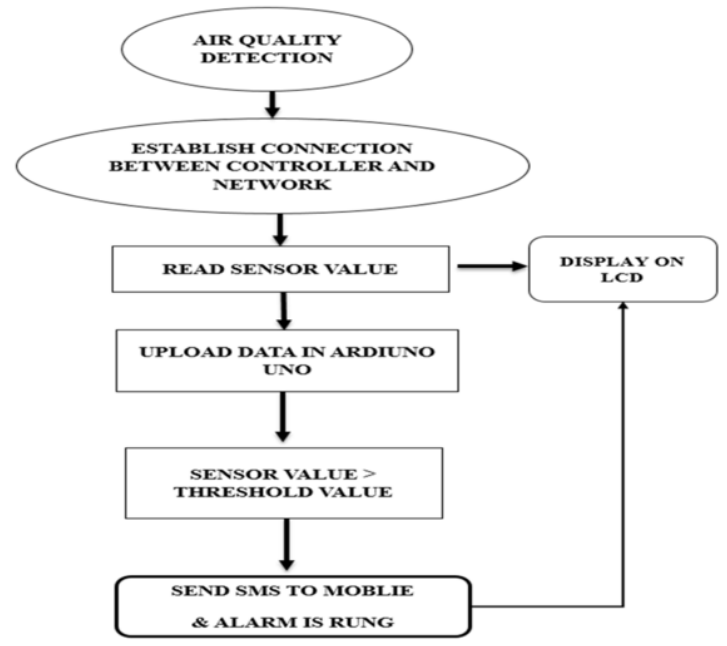

Figure 2. System Flow

As shown in Figure 2, Initially the gas sensor detects no gas. When a supply of $5 \mathrm{v}$ is passed to the arduino via a supply board. The mq-02 gas sensor is turned on. The gas sensor detects the surrounding polluted air in ppm (parts per milli). The value sensed by the sensor are analog signals when passed to arduino converts into digital values and gets displayed in the $16^{*} 2$ LCD. The Arduino checks some threshold limits set by the user to indicate the safety level of air around them [13]. When the value goes beyond a certain threshold limit which is set as the most toxic level at this stage the arduino triggers some remedial measures and also sends the user the location, level toxicity where the device is set up temperature and humidity via sms [14]. An application is also set up so that the user can get an update of the surroundings temperature, humidity and gas values from any location as the values get uploaded to a web server [15].

\section{Electronic Image File}

This proposed system contains two sections. There are Transmission and shelter gas valve control sections are shown in Figure 3 and Figure 4 respectively. The temperature and gas sensor is interfaced with a microcontroller so as to form the smart system. Where the sensors connected with the microcontroller senses the quality of gas in ppm, temperature and humidity values and updates the IoT webserver. When the gas sensor reaches the edge level then a signal is sent to the gas valve system via WSN. At 
the time it suddenly closed the solenoid valve and on the DC fan. Additionally, thereto we are distributing the robot mechanism in the drainage zone. a standing also will be displayed on the $16^{*} 2$ LCD.

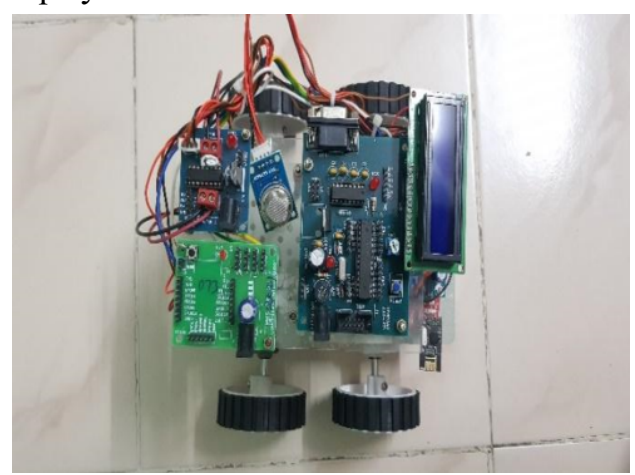

Figure 3. Aerial view of the robot

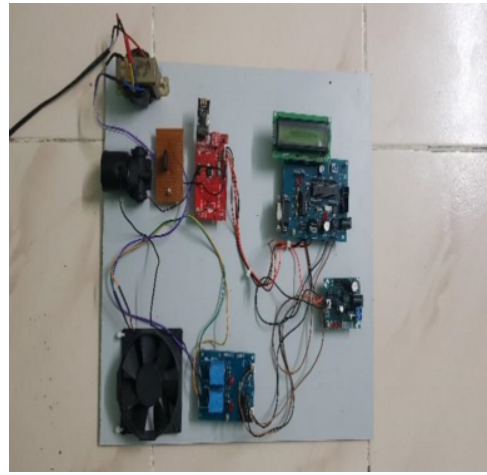

Figure 4. Aerial view of the remedial

\section{Simulation}

We used proteus to develop the simulation circuit which is used for electronic device designing in $2 \mathrm{D}$ or $3 \mathrm{D}$ format using ISIS software in the proteus. The Figure 5 and Figure 6 represents the simulated output.

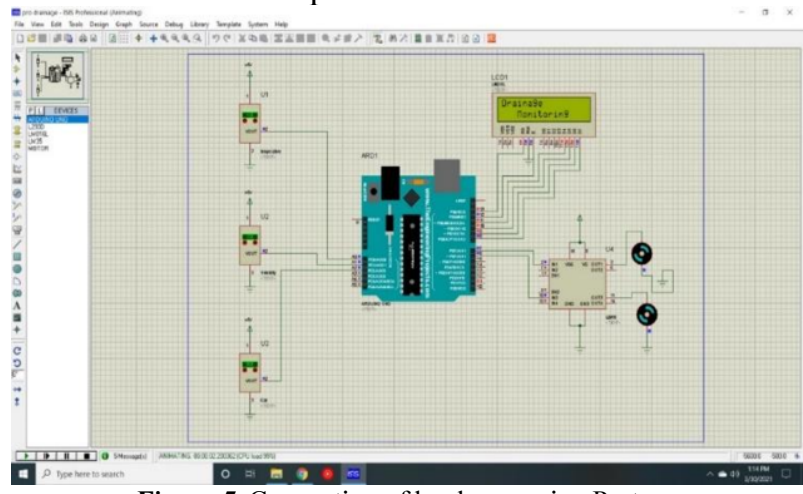

Figure 5. Connection of hardware using Proteus

In Figure 3, the initial values of the sensors are displayed in the LCD.

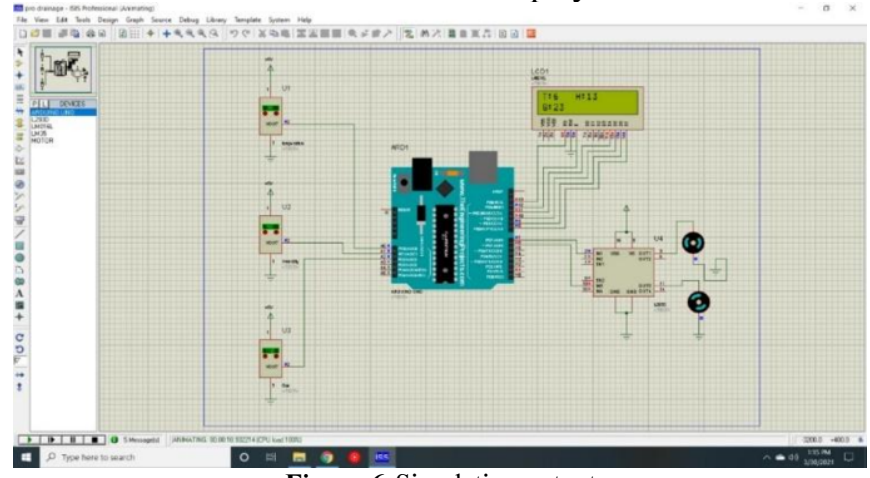

Figure 6. Simulation output 
In Figure 6, while changing the sensor values it sends the analog signal to the microcontroller which converts the analog signal to digital signal and displays the changed values in the LCD module. And at the same time relay operates the DC fan and solenoid valve simultaneously.

\section{Conclusion}

In this paper, we have come up with a potent solution to the various problems such as life threat due to poisonous gas inhalation by manhole workers, presence of toxic gases in any location of industries, no proper pollution level monitoring and indication and other numerous problems due to air pollution. Here we have proposed an IOT based smart monitoring and fast intimation of presence of toxic gases using Arduino controller. The hardware is designed not only to display the information using LCD display but also to transmit the real time data to the web at a very high baud rate efficiently. The hardware working is tested with the different injected toxic gases and the result is found to be very precise, accurate and fast.

\section{References}

[1] Jabbar, W. A., Shang, H. K., Hamid, S. N. I. S., Almohammedi, A. A., Ramli, R. M., \& Ali, M. A. H. (2019). IoT-BBMS: Internet of Things-Based Baby Monitoring System for Smart Cradle. IEEE Access, 7, 93791-93805. https://doi.org/10.1109/ACCESS.2019.2928481

[2] Lazaro, A., Boada, M., Villarino, R., \& Girbau, D. (2019). Battery-Less Smart Diaper Based on NFC Technology. IEEE Sensors Journal, 19(22), 10848-10858. https://doi.org/10.1109/JSEN.2019.2933289

[3] Berrezueta-Guzman, J., Pau, I., Martin-Ruiz, M. L., \& Maximo-Bocanegra, N. (2020). Smart-Home Environment to Support Homework Activities for Children. IEEE Access, 8, 160251-160267. https://doi.org/10.1109/ACCESS.2020.3020734

[4] Gahlot, S., Reddy, S. R. N., \& Kumar, D. (2019). Review of smart health monitoring approaches with survey analysis and proposed framework. IEEE Internet of Things Journal,6(2), 2116-2127. https://doi.org/10.1109/JIOT.2018.2872389

[5] Hesse, N., Pujades, S., Black, M. J., Arens, M., Hofmann, U. G., \& Schroeder, A. S. (2020). Learning and Tracking the 3D Body Shape of Freely Moving Infants from RGB-D sequences. IEEE Transactions on Pattern Analysis and Machine Intelligence, 42(10), 2540-2551. https://doi.org/10.1109/TPAMI.2019.2917908

[6] Gutiérrez, S. T., Fuentes, C. I., \& Díaz, M. A. (2020). Introducing SOST: An ultra-low-cost star tracker concept based on a raspberry pi and open-source astronomy software. IEEE Access, 8, 166320-166334. https://doi.org/10.1109/ACCESS.2020.3020048

[7] Hu, D., Zhang, H., Wu, Z., Wang, F., Wang, L., Smith, J. K., ... Shen, D. (2020). DisentangledMultimodal Adversarial Autoencoder: Application to Infant Age Prediction with Incomplete Multimodal Neuroimages. IEEE Transactions on Medical Imaging,39(12), 4137-4149. https://doi.org/10.1109/TMI.2020.3013825

[8] Liu, L., Li, W., Wu, X., \& Zhou, B. X. (2019). Infant cry language analysis and recognition: An experimental approach. IEEE/CAA Journal of Automatica Sinica,6(3), 778-788. https://doi.org/10.1109/JAS.2019.1911435

[9] Li, C., Pourtaherian, A., Van Onzenoort, L., Tjon, W. E., \& De With, P. H. N. (2020). Infant Monitoring System for Real-Time and Remote Discomfort Detection. IEEE Transactions on Consumer Electronics, 66(4), 336-345. https://doi.org/10.1109/TCE.2020.3031359

[10] Jiang, L., \& Hassanipour, F. (2020). Bio-Inspired Breastfeeding Simulator (BIBS): A Tool for Studying the Infant Feeding Mechanism. IEEE Transactions on Biomedical Engineering, 67(11), 3242-3252. https://doi.org/10.1109/TBME.2020.2980545

[11] R. G. Babu, V. V. Nathan, J. Bino, C. Amali and S. Ganesh, "IoT Security Enhancement with Automated Identification Device using IOT SENTINEL," 2021 11th International Conference on Cloud Computing, Data Science \& Engineering (Confluence), 2021, pp. 518-523, https://doi.org/ 10.1109/Confluence51648.2021.9377165 